\title{
El impacto psicosocial de la violencia en San Salvador
}

\author{
José Miguel Cruz'1
}

RESUMEN En este estudio se busca lograr dos objetivos: primero, describir los niveles de victimización de la población salvadoreña a causa de la violencia delictiva y el tipo de población más afectada por ella; segundo, averiguar si estos niveles de victimización están relacionados con la presencia de normas, actitudes y comportamientos que favorecen la aparición de la violencia. Para ello se utilizaron los datos del proyecto ACTIVA de El Salvador, que fue realizado por el Instituto Universitario de Opinión Pública entre los meses de octubre y noviembre de 1996 con una muestra de 1290 entrevistas personales, presuntamente representativa de las personas entre los 18 y 70 años de edad que residen en el Área Metropolitana de San Salvador. La muestra se obtuvo mediante un muestreo probabilístico y multietápico. Los resultados revelan que el nivel de victimización por la violencia es bastante alto en San Salvador y que afecta sobre todo a los hombres y a los jóvenes, y sugieren que las personas que han sido víctimas de agresiones graves suelen mostrar con más frecuencia que el resto normas de justificación y aprobación del uso de la violencia, inclinación por el uso de armas, y conductas de agresión hacia otras personas.

Sin duda, la violencia constituye actualmente uno de los problemas más graves de las sociedades actuales. Ha alcanzado una magnitud muy preocupante en todo el mundo, sobre todo después de la Segunda Guerra Mundial, cuando su frecuencia comenzó a aumentar de forma notable (1) y dejó de ser un asunto de dimensión política y sociológica para convertirse en un reto para la salud pública. En varias sociedades, la violencia, tanto intencional como no intencional, se ha convertido en una de las principales causas de muerte entre la población joven, y ello ha generado campañas para ha-

\footnotetext{
1 Instituto Universitario de Opinión Pública, Universidad Centroamericana José Simeón Cañas, teléfono: (503) 273-4400, ext. 417; fax: (503) 2737020,e-mail: mcruz@iudop.uca.edu.sv
}

cerle frente con urgencia. La investigación que se describe en este artículo forma parte de ese esfuerzo.

Una de las regiones más afectadas por el problema de la violencia es la de América Latina y el Caribe. De acuerdo con el Banco Mundial y la Organización Panamericana de la Salud (OPS) $(2,3)$, la Región de las Américas es la zona más violenta del mundo. En ella, las tasas de homicidios se aproximan a 20 muertes por 100000 habitantes al año. Sería erróneo pensar, sin embargo, que la violencia de semejante magnitud sea uniforme en América Latina. En años recientes se ha podido constatar que en ciertos países, como Colombia, El Salvador y Trinidad y Tabago, se registran las tasas de homicidios más elevadas de la Región (4). En el caso concreto de El Salvador, lo anterior significa que este país es uno de los que tienen mayores índices de violencia en una Región que se considera muy violenta.

Aunque a causa de las deficiencias de los registros oficiales no existe un acuerdo definitivo sobre la magnitud de la violencia en El Salvador, las investigaciones más recientes sobre el tema indican que las tasas de homicidios en este país oscilan en alrededor de 117 por 100000 habitantes (5). Ello coloca a El Salvador en una situación más grave que la de Colombia en relación con la violencia. No es propósito de este estudio intentar explicar este fenómeno, pero valga decir que el caso salvadoreño reviste características muy especiales, no solo por la aguda expresión de la violencia, sino también por sus precedentes históricos, por cuanto en ese país se libró una guerra civil en la pasada década.

Los informes de la OPS revelan que en los años setenta El Salvador presen- 
taba tasas de homicidio más elevadas que cualquier otro país americano, incluso superiores a las de Colombia y Nicaragua (6). En la década de los ochenta, las tasas aumentaron como consecuencia de una guerra civil que duró 12 años y que dejó alrededor de 75000 muertos y tasas de mortalidad intencional rayanas en 130 por 100000 habitantes. No obstante, el fin de la guerra no se tradujo en una reducción de estas tasas; por el contrario, existen datos que sugieren que el fenómeno se consolidó y adoptó otras expresiones, la más visible de las cuales es la delincuencia. En los sondeos de opinión pública se ha registrado que, desde 1993, alrededor de $25 \%$ de los salvadoreños habían sido víctimas, directa o indirectamente, de algún acto delictivo $(7,8)$, lo cual ejerció un impacto decisivo en la sociedad de El Salvador. Después de años en los cuales los salvadoreños se mostraban preocupados por la economía y la guerra, a partir de 1992 - cuando se firmaron los acuerdos de paz que finalizaron con el conflicto bélico- la violencia delictiva comenzó a considerarse el principal problema de orden público. Un sondeo de opinión realizado en 1996 puso de manifiesto que $64,4 \%$ de los salvadoreños pensaban que el crimen era el principal problema nacional (9). En este contexto, la población comenzó a exigir medidas más drásticas para combatir la criminalidad y apoyó masivamente la aprobación de proyectos de ley que endurecían los castigos hacia los sospechosos, simplificaban los procesos judiciales y reinstauraban la pena de muerte para ciertos delitos. Simultáneamente, los sondeos de opinión pública mostraron que un sector importante de la población estaba dispuesta a ir más allá para conseguir seguridad. A mediados de 1996, casi una cuarta parte de los salvadoreños coincidían en que "algunas veces es mejor no hacer caso a la ley y resolver los problemas sin esperar soluciones legales", y un porcentaje similar apoyaba la idea de que "la gente tiene el derecho de buscar la justicia por su propia mano" (10). Los sondeos no permitían confirmar los efectos del crimen y de las experiencias de victimización en esas opiniones (ni se dis- pone de datos de años anteriores cuando la delincuencia no era un problema tan agudo), pero reflejaban actitudes que de algún modo pueden allanar el camino hacia el uso de la violencia en la actualidad.

Prescindiendo del análisis de lo que puede ser o no la violencia, en el presente estudio se adoptó la definición propuesta por el equipo de investigadores del proyecto ACTIVA. Por violencia se entiende el uso de la fuerza física o verbal o la amenaza del uso de la fuerza física para producir daños a una persona o grupo (11). En tal sentido, la victimización sería el acto en el cual una persona es objeto del uso de esa fuerza y la que le produce un daño físico o psicológico.

Con más frecuencia, y dependiendo del interés de los investigadores, los efectos de la violencia se han estudiado sobre la base de las secuelas físicas y psicológicas que genera un acto violento. De hecho, según las revisiones de estudios sobre la violencia, se conoce más sobre la víctima que sobre el victimario, aunque los estudios muestran la cercanía demográfica de ambos grupos $(12,13)$. Pero la violencia posee una característica consustancial que le permite reproducirse una vez que aparece. El conocido adagio "la violencia engendra violencia" pone al descubierto el hecho de que una consecuencia de este fenómeno es también su reproducción. Como afirmó Martín-Baró, "la agresión desencadena un proceso que, una vez puesto en marcha, tiende a incrementarse sin que para detenerlo baste con conocer sus raíces" (14). Al hilo de este razonamiento, varios estudios han mostrado cómo los niños que han sido víctimas repetidas de violencia en el hogar tienen mayor probabilidad de desarrollar conductas agresivas cuando son adultos que los que no han sido maltratados $(15,16)$. Pero, además de esos enfoques que subrayan las consecuencias de la violencia provocada en entornos primarios y por agentes vinculados afectivamente con las víctimas, en los últimos años, y con la rúbrica de los estudios de sociedades que enfrentan niveles epidémicos de violencia, se ha configurado una interpretación sobre el impacto de la violencia relacionada con los sistemas simbólicos y normativos que permiten o reproducen la violencia como producto de una sociedad que se percibe a sí misma como victimizada (17). Esto es lo que conforma lo que en adelante se denominará impacto psicosocial de la violencia.

Cada vez es mayor la conciencia de que el crimen - no solo en su dimensión personal y familiar sino también en la social一, al afectar intensamente a amplios grupos sociales, puede permitir la aparición de actitudes y normas que, buscando valores de seguridad ciudadana, estimulan conductas de defensa capaces de convertirse fácilmente en agresión. Estas erosionan la percepción de la institucionalidad vigente y promueven a un tiempo las medidas de orden individual o privado que rompen el acuerdo social.

Todo ello pone de relieve la experiencia de victimización del crimen en una sociedad que se ve desbordada por el mismo. A este nivel, ya no es posible pensar que un acto criminal sufrido por una persona vaya a acarrear solamente consecuencias físicas y psicológicas en la víctima. Por el contrario, es probable que dicho acto ejerza efectos que cristalicen socialmente en las relaciones del sujeto con su entorno bajo la construcción de nuevas normas sociales basadas en el miedo y la desconfianza. Ello puede contribuir al pernicioso ciclo de generación de la violencia en un contexto en el cual -a diferencia de las guerras y los conflictos civiles- la violencia criminal carece de rostro concreto y puede aparecer en cualquier parte.

Así, en el contexto salvadoreño actual se subraya la necesidad de estudiar las experiencias de victimización de los ciudadanos, no tanto en el ámbito de los costos físicos y psicológicos para la víctima, sino en cuanto al impacto psicosocial - esto es, la construcción de actitudes, normas y pautas conductuales- que permitirá que la violencia se reproduzca en la sociedad. Por tanto, este trabajo, basado en los datos del estudio ACTIVA en San Salvador, busca, por un lado, establecer una medida de la victimización causada por la violencia criminal en el 
Área Metropolitana de San Salvador (AMSS), describiendo, además, los grupos demográficos que suelen ser afectados y, por otro, conocer la asociación entre los distintos niveles de victimización y algunas normas, actitudes y pautas de comportamiento que tratan de justificar y favorecen la violencia en distintas circunstancias. En este estudio se parte del supuesto de que una persona que haya sido víctima de la violencia en el AMSS tendrá actitudes, normas y conductas que promoverán el uso de violencia en mayor medida que aquellas que no hayan tenido experiencias de victimización.

\section{MATERIALES Y MÉTODOS}

Siguiendo un muestreo probabilístico, se realizaron 1290 entrevistas válidas a personas de ambos sexos entre 18 y 70 años de edad y residentes en el AMSS, la concentración urbana más grande del país, que está conformada por 11 municipios. La distribución de los integrantes de la muestra por sexo y edad fue muy similar a la distribución poblacional registrada por el V Censo de Población (18); sin embargo, para probar que la muestra provenía de la población, se decidió aplicar la prueba $t$ de Student para cada tramo de edad. Los resultados de la prueba indican que en todos los tramos, exceptuando dos, las diferencias entre la población y la muestra no fueron estadísticamente significativas $(P>0,05) \mathrm{y}$, por lo tanto, provienen de la misma población (cuadro 1). Se encontró que las mujeres de 45 a 49 años estaban sobrerrepresentadas en la muestra, al igual que los hombres de 50 a 70 años. En el primer caso se decidió preservar la muestra, porque se consideró que el valor de $t$ estaba justificado por el pequeño número de casos. En el caso segundo, se decidió ajustar los pesos de los tres grupos de edad sobrerrepresentados (50, 54 y 60 años), por un peso de 0,5 , y los restantes quedaron con su peso original (1). La muestra final del estudio estuvo de este modo integrada por 1269 casos.

El procedimiento de selección de la muestra se realizó en tres etapas con objeto de obtener al menos 1200 entrevistas. Teniendo presentes los posibles rechazos y un número indeterminado de entrevistas no realizables por otras causas, se decidió visitar 1600 hogares para evitar procedimientos de sustitución arbitraria. En primer lugar, se escogió al azar una muestra sistemática de los 893 segmentos censales en que está dividida el AMSS, con una probabilidad de selección proporcionada al tamaño poblacional. No se eligió el estrato económico como criterio de selección. Así, se seleccionaron 111 segmentos. A continuación, y a fin de calcular el número de viviendas que debían visitarse por segmento, esa cifra se divi- dió por el total de viviendas contenidas en los 111 segmentos (38 000), y el resultado se multiplicó por 1 600, número de viviendas que se debían visitar. La selección de las viviendas se llevó a cabo por medio de una selección sistemática con arranque aleatorio. Para cumplir con la tercera etapa, esto es, la elección de las personas que habrían de entrevistarse en cada vivienda, se usó una tabla en la cual se recogían los datos de las personas que vivían en ella. Cada línea de dicha tabla representaba un sujeto. La tabla contenía asimismo marcas en intervalos de tres líneas, que señalaban los sujetos que debían ser entrevistados si cumplían los requisitos prefijados: ser inquilino de la vivienda y tener entre 18 y 70 años de edad.

El cuestionario que se utilizó en el presente estudio fue el del Proyecto ACTIVA, que está formado por una tabla demográfica y varias escalas que miden diversos aspectos relacionados con la violencia. En el caso salvadoreño, se añadieron algunos reactivos de interés local: ingresos y gastos de la familia del entrevistado, opciones políticas de los entrevistados, participación como combatiente en la guerra civil, etc.

En el presente trabajo se utilizaron variables construidas a partir de algunos apartados (item) y escalas del instrumento. Seguidamente, se describen dichas variables. El nivel de victimiza-

CUADRO 1. Distribución de la población y de la muestra según el sexo y los grupos de edad, San Salvador, 1996. Proyecto ACTIVA

\begin{tabular}{|c|c|c|c|c|c|c|c|c|c|c|c|c|}
\hline \multirow{2}{*}{$\begin{array}{l}\text { Grupo } \\
\text { de edad }\end{array}$} & \multicolumn{6}{|c|}{ No. } & \multicolumn{6}{|c|}{ Porcentaje } \\
\hline & Hombre & Mujer & \multicolumn{2}{|c|}{ Hombre } & \multicolumn{2}{|c|}{ Mujer } & Hombre & Mujer & \multicolumn{2}{|c|}{ Hombre } & \multicolumn{2}{|c|}{ Mujer } \\
\hline $18-24$ & 87772 & 107942 & 125 & 125 & 143 & 143 & 25,4 & 25,7 & 21,7 & 22,5 & 20,0 & 20,0 \\
\hline $25-29$ & 57362 & 69065 & 75 & 75 & 100 & 100 & 16,6 & 16,5 & 13,0 & 13,5 & 14,0 & 14,0 \\
\hline $40-44$ & 31057 & 34876 & 48 & 48 & 84 & 84 & 9,0 & 8,3 & 8,3 & 8,7 & 11,8 & 11,8 \\
\hline $45-49$ & 23254 & 25848 & 62 & 62 & 61 & 61 & 6,7 & 6,2 & 10,8 & 11,2 & 8,5 & 8,5 \\
\hline $50-70$ & 63118 & 82673 & 116 & 95 & 137 & 137 & 18,3 & 9,7 & 20,1 & 17,0 & 19,2 & 19,2 \\
\hline Total & 345337 & 419847 & 576 & 555 & 714 & 714 & 45,1 & 54,9 & 44,7 & 43,7 & 55,3 & 56,3 \\
\hline
\end{tabular}

${ }^{a} R=$ real.

${ }^{b} \mathrm{P}=$ ponderado. 
ción es una variable construida a partir de la combinación de ocho apartados de victimización: robo a mano armada, amenaza para obtener dinero, amenaza para cambiar de residencia, golpes, herido con arma blanca, herido con arma de fuego, secuestro y asesinato de un pariente. No se incluyeron los apartados que recogen exposición a la violencia (vio un robo, vio a alguien siendo herido), ni los referidos a victimización en manos de la policía (maltrato y extorsión por parte de la policía), ni el correspondiente al suicidio, por cuanto se consideró que hacen referencia a una dimensión distinta de la victimización delictiva. Dado que cada experiencia de victimización es diferente, es decir, tiene un impacto distinto en la víctima (no es lo mismo un robo que un secuestro), se decidió ponderar de forma empírica cada tipo de agresión sufrida a partir de una proporción inversamente proporcional a la frecuencia con que se produce. Así, el delito más frecuente tendría un valor igual a 1, y, a medida en que disminuía la frecuencia de los otros delitos, su peso era mayor. Teniendo en cuenta esas ponderaciones - que jerarquizan los hechos de violencia sufridos por el entrevistado-, se construyó una variable categórica única que define cuatro niveles de victimización según la gravedad y la frecuencia del acto violento, que se expresan de la siguiente manera: a) ausencia de victimización (personas que no han sufrido ninguna agresión en el lapso de un año); b) victimización leve (personas que han sido víctimas de un acto violento leve, como haber sido asaltado o amenazado a cambio de dinero); c) victimización moderada (personas que han sufrido varios actos violentos, como un asalto o golpes), y d) victimización intensa (personas que en más de una ocasión han sufrido agresiones violentas, que incluyen algunas muy graves, como heridas por arma blanca o de fuego o incluso el asesinato).

Las variables demográficas corresponden a los apartados del cuestionario que atañen al sexo, la edad y el nivel educativo. En algunos casos, los valores de estas variables se recategorizaron para facilitar los análisis.
La variable normas de respaldo del uso de la violencia está constituida por los siguientes reactivos, que exploran normas de uso de la violencia: a) Para educar a los niños es necesario el castigo físico; b) Existen situaciones en las que está justificado que un adulto le pegue a un niño que no es de él; c) Existen situaciones en las cuales se justifica que un hombre le dé una cachetada a la esposa; d) Existen situaciones en las cuales se justifica que una mujer le dé una cachetada a su esposo; e) Si una mujer ha sido infiel merece que el marido le pegue; f) Una mujer tiene el derecho de agredir a otra que le está quitando a su marido; g) Si las autoridades fallan, la gente tiene el derecho a tomar la justicia por su propia cuenta; h) La policía tiene el derecho de invadir una casa sin orden de cateo para perseguir delincuentes; i) La policía tiene el derecho de detener a jóvenes que considere sospechosos por su aspecto físico, y j) En algunos casos se justifica que la policía torture a los sospechosos para obtener información. La fiabilidad de esta variable, medida mediante la alfa de Cronbach, fue de 0,654 y la escala utilizada para medirla fue la de Likert (19). El valor menor de la escala expresa la ausencia de esas normas y el valor más alto, su presencia más clara.

La variable aprobación del uso de la violencia está formada por los siguientes reactivos: a) Si una persona se cuela y no hace fila y otra la insulta. Usted aprobaría, no aprobaría pero entendería, no aprobaría ni entendería; b) Suponga que una persona hiere seriamente a alguien que le quitó el/la esposo(a). Usted... ; c) Suponga que una persona mata a alguien que le ha violado a una hija. Usted. .. ; d) Si hay una persona que mantiene asustada a su comunidad y alguien la mata. Usted. . . , y e) Si un grupo de personas comienza a hacer limpiezas sociales. Usted. . . La confiabilidad de esta escala, medida con la alfa de Cronbach, fue de 0,785 .

La variable de inclinación por las armas está integrada por dos apartados de la escala de actitudes de ACTIVA, se midió mediante una escala de Likert, y busca medir la inclinación a usar las armas. Su confiabilidad fue elevada (alfa de Cronbach $=0,809$ ). Los dos apartados son: a) El tener un arma en casa hace que la casa sea más segura, y b) Portar un arma hace que una persona esté más segura. El valor más bajo señala que no hay ninguna inclinación por las armas, mientras que el valor más alto muestra una elevada afición por las mismas.

La variable intolerancia comunitaria, que mide actitudes de intolerancia a las diferencias en el entorno comunitario de residencia del entrevistado, se construyó con los siguientes reactivos: a) Un vecindario debería estar compuesto por personas de una misma clase social; b) ... de una misma religión; c) ... de una misma raza u origen étnico, y d) con las mismas ideas políticas. Su confiabilidad fue alta (alfa de Cronbach $=0,82$ ).

La variable nivel de agresión hacia personas particulares mide la frecuencia con que una persona admite haber agredido a otra que no forma parte de su familia en el lapso de un año. La confiabilidad de la escala con que se midió fue aceptable (alfa de Cronbach = 0,652 ). Los siguientes apartados conforman esta variable: a) Amenazó con lastimar a alguien que no era familiar suyo; b) Golpeó a alguien que no era familiar suyo, y c) Se valió de alguien para que hiciera mal a una persona. Sin embargo, la distribución de esta variable no fue gaussiana $y$, por tanto, se decidió transformarla en una escala logarítmica natural, que guardó alta correlación lineal con la variable original ( $r$ de Pearson $=0,9860$ ).

Dado que todas las escalas tenían un recorrido de valores distinto, y para facilitar el análisis de las mismas, se procedió a estandarizarlas en un mismo recorrido de valores (de 1 a 5), de tal forma que 1 representase siempre el valor más bajo de la variable y 5 , el más alto.

Veintinueve encuestadores que formaban parte del personal del Instituto Universitario de Opinión Pública fueron instruidos para administrar el cuestionario ACTIVA. Una vez capacitados, los entrevistadores fueron destinados a visitar hogares en equipos de cinco personas a cargo de un supervi- 
sor. Cada encuestador visitó un hogar de una zona asignada a su equipo. Las entrevistas se hicieron personalmente mediante visita al hogar y el encuestador tenía la obligación de visitar la vivienda hasta completar las encuestas asignadas a ella. A cada persona seleccionada se le explicaron los objetivos y el procedimiento de la entrevista. En todos los casos se entrevistó únicamente a las personas que aceptaron colaborar y que cumplieron los requisitos de la selección. Los cuestionarios se revisaron, y se excluyen del análisis tanto los cumplimentados de forma incompleta como los que contenían incongruencias. Los no concluidos a la postre en el análisis se codificaron y los datos se analizaron utilizando el paquete estadístico SPSS para Windows, versión $6.0(20,21)$.

Los análisis estadísticos realizados constan de dos partes independientes. En la primera, los valores de la variable nivel de victimización se compararon con las variables sexo, edad y nivel educativo, mediante tablas de contingencia a fin de conocer si algunos grupos de la población sufrían niveles de victimización a causa de la violencia delictiva más altos que otros. Para contrastar la hipótesis nula de que no hay diferencias en los niveles de victimización entre los distintos valores de las variables demográficas se utilizó la prueba de ji al cuadrado. Este análisis también se realizó para conocer los niveles de victimización criminal que soportaban los encuestados.

La segunda parte del análisis responde a los objetivos centrales de este artículo y busca averiguar si el nivel de victimización a causa del crimen está relacionado con normas, actitudes y pautas conductuales que promueven el uso de la violencia en otros entornos. En concreto, se buscó averiguar si el aumento de la experiencia de victimización estaba relacionado con un valor más elevado en la escala de normas que justifican y hacen posible el uso de la violencia, con una mayor aprobación hacia el uso de la violencia, con una inclinación más marcada por el uso de las armas, con una actitud más intensa de intolerancia comunitaria o con el aumento de pautas de conducta agresivas hacia personas particulares. Las variables de normas de respaldo del uso de la violencia y de aprobación al uso de la violencia se refieren más al ámbito normativo de las conductas sociales, las variables de inclinación por las armas y de intolerancia comunitaria aluden más a elementos actitudinales, y la variable de agresión hacia personas particulares subraya pautas conductuales de violencia. Para ello, la variable de nivel de victimización se utilizó como variable independiente y todas las restantes, como variables dependientes. La relación entre la primera variable y las restantes se analizó de forma separada para cada una de las variables mediante el análisis de la varianza. La variable nivel de agresión hacia personas particulares se transformó a una escala de logaritmos neperianos por no distribuirse con arreglo a la curva de Gauss. Dada la elevada correlación lineal entre las dos variables, esta variable se codificó como ordinal. Para cada una de las variables dependientes, la hipótesis nula utilizada fue que las medias de las variables no diferían según los distintos niveles de victimización sufridos por la persona. El nivel de significación estadística alfa adoptado fue de 0,05.

\section{RESULTADOS}

En el cuadro 2 se presentan los valores de la variable nivel de victimización según las variables demográficas analizadas en este estudio. Se estima que $59,1 \%$ de la población estudiada no ha sufrido ninguna agresión delictiva en el lapso de un año, $22,4 \%$ ha padecido agresiones que pueden conside-

CUADRO 2. Porcentajes de victimización (e intervalos de confianza de 95\%) de los residentes del Área Metropolitana de San Salvador, según las variables demográficas analizadas en este estudio. San Salvador, 1996. Proyecto ACTIVA

\begin{tabular}{|c|c|c|c|c|}
\hline \multirow{2}{*}{$\begin{array}{c}\text { Variables } \\
\text { demográficas }\end{array}$} & \multicolumn{4}{|c|}{ Nivel de victimización } \\
\hline & Ninguno & Leve & Moderado & Intenso \\
\hline Todos & $\begin{array}{c}59,1 \\
(56,4-61,8)\end{array}$ & $\begin{array}{c}22,4 \\
(20,1-24,7)\end{array}$ & $\begin{array}{c}12,8 \\
(11,0-14,6)\end{array}$ & $\begin{array}{c}5,6 \\
(4,3-6,9)\end{array}$ \\
\hline \multicolumn{5}{|l|}{ Sexo } \\
\hline Masculino & $\begin{array}{c}57,1 \\
(53,1-61,2)\end{array}$ & $\begin{array}{c}21,0 \\
(17,6-24,4)\end{array}$ & $\begin{array}{c}15,9 \\
(12,9-18,9)\end{array}$ & $\begin{array}{c}6,0 \\
(4,1-7,9)\end{array}$ \\
\hline Femenino & $\begin{array}{c}60,6 \\
(57,0-64,2)\end{array}$ & $\begin{array}{c}23,5 \\
(20,4-26,6)\end{array}$ & $\begin{array}{c}10,5 \\
(8,3-12,7)\end{array}$ & $\begin{array}{c}5,3 \\
(3,7-6,9)\end{array}$ \\
\hline \multicolumn{5}{|l|}{ Edad (años) } \\
\hline $18-25$ & $\begin{array}{c}51,5 \\
(45,9-57,1)\end{array}$ & $\begin{array}{c}24,6 \\
(19,8-29,4)\end{array}$ & $\begin{array}{c}16,7 \\
(12,5-20,9)\end{array}$ & $\begin{array}{c}7,2 \\
(4,3-10,4)\end{array}$ \\
\hline $26-40$ & $\begin{array}{c}57,1 \\
(52,9-61,3)\end{array}$ & $\begin{array}{c}24,0 \\
(20,3-27,7)\end{array}$ & $\begin{array}{c}12,0 \\
(9,2-14,8)\end{array}$ & $\begin{array}{c}6,9 \\
(4,7-9,1)\end{array}$ \\
\hline $41-55$ & $\begin{array}{c}64,1 \\
(58,6-69,6)\end{array}$ & $\begin{array}{c}20,2 \\
(15,6-24,8)\end{array}$ & $\begin{array}{c}12,5 \\
(8,7-16,3)\end{array}$ & $\begin{array}{c}3,2 \\
(1,2-5,2)\end{array}$ \\
\hline$\geq 56$ & $\begin{array}{c}72,1 \\
(64,8-79,4)\end{array}$ & $\begin{array}{c}16,7 \\
(10,7-22,7)\end{array}$ & $\begin{array}{c}8,5 \\
(4,0-13,0)\end{array}$ & $\begin{array}{c}2,7 \\
(0,1-5,3)\end{array}$ \\
\hline \multicolumn{5}{|l|}{ Nivel educativo } \\
\hline Ninguno & $\begin{array}{c}76,0 \\
(67,1-84,9)\end{array}$ & $\begin{array}{c}16,0 \\
(8,3-23,7)\end{array}$ & $\begin{array}{c}5,7 \\
(0,9-10,5)\end{array}$ & $\begin{array}{c}2,3 \\
(0-5,4)\end{array}$ \\
\hline Primaria & $\begin{array}{c}60,7 \\
(56,0-65,4)\end{array}$ & $\begin{array}{c}23,0 \\
(19,0-27,0)\end{array}$ & $\begin{array}{c}10,3 \\
(7,4-13,2)\end{array}$ & $\begin{array}{c}6,0 \\
(3,7-8,3)\end{array}$ \\
\hline Secundaria & $\begin{array}{c}54,2 \\
(49,4-58,9)\end{array}$ & $\begin{array}{c}24,1 \\
(20,0-28,2)\end{array}$ & $\begin{array}{c}16,2 \\
(12,6-19,8)\end{array}$ & $\begin{array}{c}5,6 \\
(3,4-7,8)\end{array}$ \\
\hline Técnico & $\begin{array}{c}62,2 \\
(49,5-74,9)\end{array}$ & $\begin{array}{c}18,0 \\
(7,9-28,1)\end{array}$ & $\begin{array}{c}15,3 \\
(5,9-24,7)\end{array}$ & $\begin{array}{c}4,5 \\
(0-9,9)\end{array}$ \\
\hline Universitario & $\begin{array}{c}58,9 \\
(53,1-69,7)\end{array}$ & $\begin{array}{c}21,6 \\
(16,8-26,4)\end{array}$ & $\begin{array}{c}13,0 \\
(9,1-16,9)\end{array}$ & $\begin{array}{c}6,4 \\
(3,5-9,3)\end{array}$ \\
\hline
\end{tabular}


rarse victimización leve, $12,8 \%$ son víctimas moderadas, y 5,6\%, han sufrido victimización intensa. Si se suman los porcentajes de victimización de leve a intensa, se concluye que $40,9 \%$ de los entrevistados habían sido víctimas de algún hecho violento de tipo delictivo.

Sin embargo, los resultados muestran que el nivel de victimización no fue el mismo para distintos grupos demográficos. En los hombres, los niveles de victimización fueron un poco más altos e intensos que en las mujeres (ji al cuadrado $=8,83 ; \mathrm{gl}=3 ; P<0,01$ ). Los entrevistados más jóvenes (entre 18 y 26 años) solían ser víctimas de agresiones con más frecuencia que las personas de cualquier otro grupo de edad $\mathrm{y}$, a medida que aumenta la edad, los niveles de victimización disminuyen notablemente (ji al cuadrado $=26,87 ; \mathrm{gl}=9 ; P<0,01)$. Como se observa en el cuadro 2, las diferencias entre los niveles de victimización según el grado de escolaridad no fueron estadísticamente significativas (ji al cuadrado $=20,66 ; \mathrm{gl}=12 ; P>0,05$ ).

En el cuadro 3 aparecen las medias de las variables normativas, actitudinales y conductuales según los distintos niveles de victimización sufridos por los ciudadanos. Como puede observarse, los valores de dichas variables aumentan, a excepción de la intolerancia comunitaria, a medida que lo hacen los niveles de victimización delictiva. Las diferencias entre las medias no fueron estadísticamente significativas en el análisis de la varianza. Sin embargo, esto no implica que en todas esas escalas (normas de apoyo al uso de la violencia, aprobación del uso de la violencia, inclinación por las armas $\mathrm{y}$ agresiones a particulares) las diferencias entre cada uno de los niveles de victimización fuesen significativas. De hecho, los análisis post-hoc realizados usando el procedimiento de la diferencia significativa más pequeña (least significant difference) mostraron que en la mayoría de los casos el grupo de personas que habían sufrido victimización intensa fue distinto de los restantes, es decir, que las variaciones principales se encontraban entre quienes formaban el grupo de víctimas agredidas gravemente y los grupos restantes.

CUADRO 3. Medias (y sus correspondientes intervalos de confianza de 95\%) de las variables normativas, actitudinales y conductuales, según nivel de victimización. San Salvador, 1996. Proyecto ACTIVA

\begin{tabular}{lccccc}
\hline & \multicolumn{5}{c}{ Variables $^{\mathrm{a}}$} \\
\cline { 2 - 6 } $\begin{array}{c}\text { Nivel de } \\
\text { victimización }\end{array}$ & $\begin{array}{c}\text { Normas de } \\
\text { respaldo del uso } \\
\text { de la violencia }\end{array}$ & $\begin{array}{c}\text { Aprobación del } \\
\text { uso de la } \\
\text { violencia }\end{array}$ & $\begin{array}{c}\text { Tendencia } \\
\text { a usar } \\
\text { armas }\end{array}$ & $\begin{array}{c}\text { Intolerancia } \\
\text { comunitaria }\end{array}$ & $\begin{array}{c}\text { Agresión hacia } \\
\text { personas } \\
\text { particulares }\end{array}$ \\
\hline Todos & 1,56 & 2,72 & 1,75 & 1,85 & 1,11 \\
Ninguna & $(1,53-1,59)$ & $(2,66-2,78)$ & $(1,69-1,82)$ & $(1,78-1,91)$ & $(1,09-1,14)$ \\
& 1,55 & 2,63 & 1,71 & 1,85 & 1,07 \\
Leve & $(1,51-1,59)$ & $(2,55-2,71)$ & $(1,62-1,79)$ & $(1,77-1,94)$ & $(1,05-1,10)$ \\
& 1,55 & 2,74 & 1,76 & 1,73 & 1,10 \\
Moderada & $(1,49-1,61)$ & $(2,62-2,85)$ & $(1,62-1,91)$ & $(1,60-1,86)$ & $(1,05-1,15)$ \\
& 1,55 & 2,86 & 1,73 & 2,00 & 1,20 \\
Intensa & $(1,46-1,64)$ & $(2,71-3,01)$ & $(1,55-1,91)$ & $(1,81-2,20)$ & $(1,10-1,30)$ \\
& 1,79 & 3,22 & 2,22 & 1,91 & 1,37 \\
Valores del & $(1,63-1,95)$ & $(2,99-3,46)$ & $(1,90-2,54)$ & $(1,64-2,19)$ & $(1,19-1,55)$ \\
análisis de & $F=4,33$ & $F=8,68$ & $F=3,95$ & $F=1,97$ & $F=12,45$ \\
la varianza & $\mathrm{Gl}=3$ & $\mathrm{Gl}=3$ & $\mathrm{Gl}=3$ & $\mathrm{Gl}=3$ & $\mathrm{Gl}=3$ \\
\hline
\end{tabular}

${ }^{a}$ Todas las escalas se estandarizaron en un recorrido de valores de 1 a 5 ; donde 1 representa el valor más bajo de la variable y 5 , el valor más alto.

${ }^{\circ} \mathrm{Gl}=$ grados de libertad.

\section{DISCUSIÓN}

Los resultados del estudio ACTIVA en la capital salvadoreña revelan que el porcentaje de personas que han sufrido alguna agresión violenta de índole delictiva es bastante elevado. El hecho de que cuatro de cada 10 residentes del AMSS la hayan padecido sugiere que el problema de la violencia delictiva no se expresa ocasionalmente, sino, más bien, que posee una manifestación común, a juzgar por las estadísticas de violencia de El Salvador (5). Ello implica que buena parte de los ciudadanos salvadoreños continúan siendo víctimas de la violencia a pesar de que la guerra civil concluyó hace algunos años, con la diferencia de que este no constituye un tipo de violencia bélica.

El impacto de la violencia no solo se expresa como delitos frecuentes, sino también en su intensidad. La expresión más aguda de violencia en la capital -asesinatos, secuestros y agresiones físicas- alcanza un importante porcentaje de víctimas (alrededor de $6 \%$ ) y ello puede tener un efecto sus- tancial en la manera en que estas personas deben vivir y relacionarse con los demás en San Salvador. Los datos indican que este tipo de violencia de tipo delictivo no afecta del mismo modo a todos los ciudadanos del AMSS. A pesar de que los elevados niveles de victimización se encuentran en la mayoría de los grupos, el estudio ha arrojado pruebas de que los hombres y los jóvenes suelen ser víctimas de este tipo de violencia con más frecuencia y con mayor intensidad que las mujeres o que cualquier otro grupo de personas. Estas tendencias relativas al sexo y la edad de los afectados corresponden a la distribución demográfica de las víctimas que son objeto con mayor frecuencia de delitos, como el homicidio, según los registros del Instituto de Medicina Legal y otras fuentes de información.

Sin embargo, con los datos disponibles no es posible confirmar la existencia de una relación entre la intensidad de la violencia sufrida y la escolaridad de los ciudadanos. Las personas que viven en el AMSS afrontan la violencia social de la misma intensidad al 
margen de sus niveles de formación académica.

El hallazgo principal de esta investigación acaso sea el hecho de que la intensidad de la victimización puede estar asociada con la manera en que los ciudadanos elaboran algunos sistemas normativos, actitudinales y conductuales que a su vez hacen posible la reproducción de la violencia. En este estudio se ha observado que una persona victimizada intensamente tiende a poseer normas que respaldan el uso de la violencia con más frecuencia que otras personas. En general, los datos no muestran que el aumento sea significativo cuando se pasa del grupo de personas no victimizadas al de las que han sufrido agresiones leves o moderadas, pero el aumento se da claramente si han sido víctimas de agresiones graves. Las personas que no han sido victimizadas pueden prestar el mismo respaldo normativo al uso de la violencia — que en sí mismo es bajo- que las que han sido agredidas leve o moderadamente. Empero, cuando se ha tenido una experiencia violenta intensa, las respuestas normativas que justifican y respaldan la violencia se encuentran con mayor frecuencia. En este sentido, la gente puede percibir con más normalidad las manifestaciones de la violencia. Así, es más probable que una persona que ha sido víctima de una agresión grave esté más dispuesta a consentir su uso que las que no han sido victimizadas o que aquellas cuya victimización no haya sido tan traumática. Lo mismo sucede con ciertas actitudes concretas relacionadas con el uso de la violencia. En el estudio se encontró que la tendencia a usar armas puede estar vinculada también con el nivel de victimización sufrido, especialmente si tal experiencia de victimización ha sido muy intensa. Lo anterior significa que las personas que han sido intensamente agredidas son más proclives a usar armas que las que no lo han sido o que aquellas personas cuyas experiencias de victimización no han sido tan graves. Sin embargo, cuando se trata de actitudes de intolerancia comunitaria que se encuentran como base de las conductas de segregación social, no se encontraron prue- bas de que el nivel de victimización ejerza efecto alguno. En otras palabras, el haber sido víctima de la violencia criminal no está relacionado con una mayor o menor intolerancia hacia otros grupos sociales de la comunidad. Esto es importante porque sugiere que, a diferencia de los factores normativos y de las inclinaciones a usar armas, la victimización no estaría asociada con la intolerancia social.

Donde más claramente se constata el efecto de la relación con el grado de violencia sufrida es en la aparición de las conductas de agresión hacia otras personas. Aunque según el análisis posthoc los que han tenido experiencias de victimización leve se diferencian muy poco de los que no han sido víctimas de la violencia, las personas que han sido victimizadas de forma intensa y moderada sí tienden a diferenciarse claramente de las anteriores porque desarrollan algunas conductas agresivas. Los que no han sido víctimas de la violencia prácticamente no agreden a otros, pero los que han sido agredidos moderada o gravemente desarrollan esas conductas en mayor o menor medida.

La mayor parte de los datos anteriores sugieren que el nivel de violencia sufrida puede estar asociado con la construcción de algunas normas y actitudes que hacen posible la violencia en otros entornos. Sin embargo, más que postular que las experiencias de victimización intensa producen per se más violencia, se plantean dos interpretaciones que en el fondo no se excluyen entre sí: por un lado, los resultados muestran que las personas que pueden ser más violentas tienden a ser victimizadas con mayor intensidad; por otro, permiten pensar que frente a la experiencia de victimización se crean o refuerzan algunos sistemas normativos y de pautas conductuales que también favorecen la violencia. Se postula, además, que detrás de las actitudes, normas y comportamientos violentos puede existir un contexto de victimización delictiva que está asociado con aquellos factores. Esto no significa que la victimización por sí misma produzca más violencia, sino que no se puede soslayar su presencia en la construcción de los sistemas normativos que la reproducen. Esta investigación muestra que en la mayor parte de los casos, el cambio de los sistemas normativos y de las pautas de conducta no es directamente proporcional al nivel de afectación por la violencia, pues en varios casos se precisa vivir la violencia con gran intensidad para encontrar un cambio en las normas, actitudes y conductas, cuando ello es posible.

Los resultados obtenidos subrayan la importancia de las experiencias violentas en la constitución de las normas y los valores que determinan las relaciones sociales. En el contexto de una sociedad con niveles elevados de violencia el principio de reproducción de la violencia se torna muy complejo, ya que se produce a través de los sistemas psicosociales que interpretan la realidad y crean normas que facilitan la violencia. Esta se reproduce por medio de un proceso de sustitución de normas, valores y comportamientos en contextos muy victimizantes, ya sea por aprendizaje o porque las víctimas tratan de evitar o controlar el riesgo de sufrir otra agresión, desarrollando - paradójicamente - condiciones que facilitan la misma violencia.

El impacto psicosocial de la violencia, expresado en la modificación de las normas, actitudes y conductas de las personas, exige la concurrencia de otros factores que no se han explorado en este estudio y que, por tanto, no es posible definir, pero que han de dilucidarse en el futuro para poder entender la reproducción de la violencia y diseñar programas de atención social. Es necesario, por añadidura, buscar formas para medir mejor el impacto social de la violencia delictiva en las sociedades en las cuales alcanza cotas de endemia.

Por otro lado, los datos disponibles permiten subrayar la importancia de los niveles de victimización en la reproducción de la violencia. En una sociedad como la salvadoreña cualquier programa de intervención que preste atención a los sistemas normativos que promueven el uso de la violencia debe, por tanto, partir del hecho de que los mismos son producto de una espiral alimentada, en parte, por las experiencias vividas por los ciudadanos - muchas veces cotidianas-, aunque aque- 
llas no se produzcan en entornos primarios. Por ello, la intervención en las personas que han sufrido la violencia intensamente no puede limitarse a atender solamente sus consecuencias físicas y psicológicas, sino también a prestar atención a los factores psicosociales que se generan como producto de la misma. Se debe asimismo trabajar en el reaprendizaje de conductas alternativas y en la elaboración de una interpretación de la realidad que combata la inseguridad ciudadana a través de la solidaridad y no a través de la desconfianza generalizada. De otra manera, la violencia nunca dejará de ser el espacio común de las relaciones sociales.

Agradecimiento. Este artículo se basa en los datos del Estudio Multicéntrico sobre Actitudes y Normas Culturales frente a la Violencia (proyecto ACTIVA), que fue realizado en ocho ciudades de América Latina y España bajo los auspicios y la coordinación de

\section{REFERENCIAS}

1. Organización Panamericana de la Salud. Sociedad, violencia y salud. Memorias de la Conferencia Interamericana sobre Sociedad, Violencia y Salud. Washington, D.C.: OPS; 1996.

2. Banco Mundial. El crimen y la violencia como problemas para el desarrollo en América Latina y el Caribe. Seminario El Desafío de la Criminalidad Urbana. Río de Janeiro: Banco Interamericano de Desarrollo; 1997.

3. Bobadilla JL, Cárdenas V, Couttolenc B, Guerrero R, Remenyi MA. Medición de los costos de la violencia. Resultados de un taller organizado por la Organización Panamericana de la Salud (OPS) y el Banco Interamericano de Desarrollo. Caracas: Organización Panamericana de la Salud; 1995.

4. Roberts D. Mortalidad por lesiones no intencionales y violencia en las Américas: libro de referencia. Washington, D.C.: Organización Panamericana de la Salud; 1997.

5. Cruz JM, González LA. Magnitud de la violencia en El Salvador. Estudios Centroamericanos ECA 1997; 2:953-966.

6. Organización Panamericana de la Salud. Las condiciones de salud en las Américas. Vol. I. Washington, D.C.: OPS; 1980.

7. Instituto Universitario de Opinión Pública. Documento especial: la delincuencia urbana.
Encuesta exploratoria. Estudios Centroamericanos ECA 1993;48:471-482.

8. Instituto Universitario de Opinión Pública. Sistema de justicia, delincuencia y corrupción. La opinión de los salvadoreños. Estudios Centroamericanos ECA 1994;49: 1057-1068.

9. Instituto Universitario de Opinión Pública. Encuesta sobre la ley de emergencia y opiniones sobre la delincuencia. San Salvador: IUDOP-UCA; 1996. [Serie de informes 54.]

10. Instituto Universitario de Opinión Pública. Las actitudes de los salvadoreños en torno a las leyes. Estudios Centroamericanos ECA 1996;51:905-920.

11. Organización Panamericana de la Salud, División de Salud y Desarrollo Humano. Actitudes y normas culturales sobre la violencia en ciudades seleccionadas de la Región de las Américas. Proyecto ACTIVA. Washington, D.C.: OPS, División de Salud y Desarrollo Humano; 1996

12. National Research Council. Understanding and preventing violence. Washington, D.C.: National Academy Press; 1993.

13. Levine FJ, Rosich KJ. Social causes of violence: crafting a science agenda. Washington, D.C.: American Sociological Association; 1996. la Organización Panamericana de la Salud. La investigación en El Salvador fue posible gracias a un convenio de cooperación con el Centro de Vídeo de la Universidad Centroamericana "José Simeón Cañas", con el financiamiento de la Comisión Europea, Célula de Derechos Humanos y Democratización en América Latina. El autor agradece a Rubí Esmeralda Arana, Giovanni Flores y David Navarro sus observaciones y su colaboración en la preparación de este artículo.
14. Martín-Baró I. Acción e ideología: psicología social desde Centroamérica. San Salvador: UCA Editores; 1983.

15. Jenkins P. Threads that link community and family violence: issues for prevention. En: Hampton RL, Jenkins P, Gullota TP. Preventing violence in America. Thousand Oaks, California: Sage Publications; 1996. pp. 33-52.

16. Straus MA. Beating the devil out of them: corporal punishment in American Families. New York: Lexington Books; 1994.

17. Ratinoff L. Delincuencia y paz ciudadana. En: Banco Interamericano de Desarrollo. Hacia un enfoque integrado de desarrollo: ética, violencia y seguridad ciudadana. Washington, D.C. BID; 1996;3-18.

18. República de El Salvador. Ministerio de Economía. Dirección General de Estadísticas y Censos. Censos nacionales. V de población y IV de vivienda. Tomo General El Salvador. San Salvador: DIGESTYC; 1995.

19. Likert R. Una técnica para medir actitudes. En: Summers GF. Medición de actitudes. México, D.F.: Trillas; 1976:182-193.

20. SPSS, Inc. Entering data with SPSS Data Entry II for the IBM PC/XT/AT and PS/2. Chicago: SPSS, Inc; 1988

21. Norusis M. The SPSS guide to data analysis for SPSS/PC+. 2nd ed. Chicago: SPSS, Inc.;1991.
ABSTRACT

\section{The psychosocial impact of violence in San Salvador}

This study had two objectives: (1) to describe the levels of victimization of Salvadorians due to criminal violence, and the population groups most affected by it; (2) to learn if these levels of victimization are related to the presence of norms, attitudes, and behaviors that encourage the occurrence of violence. For this purpose data from the ACTIVA project of El Salvador were used. The project was carried out by the University Institute of Public Opinion during October and November 1996, with a sample of 1290 personal interviews that were presumed to be representative of people between 18 and 70 years of age living in the Metropolitan Area of San Salvador. The sample was obtained through multistage probability sampling. The results show that the level of victimization from violence is quite high in San Salvador and that it especially affects adult and young men. The results also indicate that persons who have been victims of serious aggression tend to show, more frequently than average, norms justifying and approving the use of violence, a tendency to use weapons, and aggressive behaviors toward other people. 